\title{
Exploring the lower mass function in the young open cluster IC $4665^{\star, \star \star}$
}

\author{
W. J. de Wit ${ }^{1}$, J. Bouvier ${ }^{1}$, F. Palla ${ }^{2}$, J.-C. Cuillandre ${ }^{3}$, D. J. James ${ }^{4}$, T. R. Kendall ${ }^{5}$, \\ N. Lodieu ${ }^{6,8}$, M. J. McCaughrean ${ }^{7,8}$ E. Moraux ${ }^{9}$, S. Randich ${ }^{2}$, and L. Testi ${ }^{2}$
}

\author{
${ }^{1}$ Laboratoire d'Astrophysique, Observatoire de Grenoble, BP 53, 38041 Grenoble, Cedex 9, France \\ e-mail: dewit@obs.ujf-grenoble.fr \\ 2 INAF, Osservatorio Astrofisico di Arcetri, Largo E. Fermi 5, 50125 Florence, Italy \\ 3 Canada-France-Hawaii Telescope Corp., Kamuela, HI 96743, USA \\ 4 Physics and Astronomy Department, Vanderbilt University, 1807 Station B, Nashville, TN 37235, USA \\ 5 Centre for Astrophysics Research, Science \& Technology Research Institute, Department of Physics, Astronomy \& Mathematics, \\ University of Hertfordshire, College Lane, Hatfield AL10 9AB, UK \\ ${ }^{6}$ University of Leicester, Department of Physics \& Astronomy, University Road, Leicester LE1 7RH, UK \\ 7 University of Exeter, School of Physics, Stocker Road, Exeter EX4 4QL, UK \\ 8 Astrophysikalisches Institut Potsdam, An der Sternwarte 16, 1448 Potsdam, Germany \\ 9 Institute of Astronomy, Cambridge, CB3 OHA, UK
}

Received 25 August 2005 / Accepted 28 October 2005

\section{ABSTRACT}

We present a study of the young (30-100 Myr) open cluster IC 4665 with the aim to determine the shape of the mass function well into the brown dwarf regime. We photometrically select 691 low-mass stellar and 94 brown dwarf candidate members over an area of 3.82 square degrees centred on the cluster. $K$-band follow-up photometry and Two-Micron All-Sky Survey data allow a first filtering of contaminant objects from our catalogues. A second filtering is performed for the brightest stars using proper motion data provided by the Tycho- 2 and UCAC2 public catalogues. Contamination by the field population for the lowest mass objects is estimated using same latitude control fields. We fit observed surface densities of various cluster populations with King profiles and find a consistent tidal radius of $1.0^{\circ}$. The presence of possible mass segregation is discussed. In most respects investigated, IC 4665 is similar to other young open clusters at this age: (1) a power law fit to the mass function between 1 and $0.04 M_{\odot}$ results in best fit for a slope of -0.6 ; (2) a cusp in the mass function is noticed at about the substellar boundary with respect to the power law description, the interpretation of which is discussed; (3) a fraction between 10-19\% for BDs with $M \gtrsim 0.03 M_{\odot}$ to total members; (4) a best-fit lognormal function to the full mass distribution shows an average member mass of $0.32 M_{\odot}$, if IC 4665 has an age of $50 \mathrm{Myr}$.

Key words. Galaxy: open clusters and associations: individual: IC 4665 - stars: low mass, brown dwarfs stars: luminosity function, mass function - techniques: photometric

\section{Introduction}

Formation theories envisage brown dwarfs (BDs) evolving from the gravitational collapse of the smallest unities in the opacity limited fragmentation of giant molecular clouds like stars, e.g. Shu et al. (1987). Otherwise they may form from the condensation of solids within circumstellar (or circumbinary)

* Based on observations obtained at the Canada-France-Hawaii Telescope (CFHT) which is operated by the National Research Council of Canada, the Institut National des Science de l'Univers of the Centre National de la Recherche Scientifique of France, and the University of Hawaii.

$\star \star$ Table 3 is only available in electronic form at the CDS via anonymous ftp to cdsarc.u-strasbg.fr $(130.79 .128 .5)$ or via http://cdsweb.u-strasbg.fr/cgi-bin/qcat?J/A+A/448/189 disks like planets (Lin et al. 1998; Papaloizou \& Terquem 2001; Jiang et al. 2004). Innovative ideas let BDs undergo a starlike gravitational collapse initially, but they terminate the subsequent mass accretion on the formed hydrostatic core. This could be due to the removal of this stellar seed from the material reservoir after a dynamical interaction with one of its siblings (Reipurth \& Clarke 2001). Conversely the growth of the stellar seed can be halted by the removal of the material reservoir itself either due to the intense radiation pressure from nearby young high-mass stars (Kroupa 2001; Whitworth \& Zinnecker 2004) or due to the actual collision of protostars, by which the impact causes a large portion of the envelope to be removed. As such these various ideas supply testable observational predictions like "BD halos" of young clusters, a relative proximity to massive stars or a regular mixture with low mass 
stars. They will lead to an increased understanding of BDs and the star formation (SF) process in general.

Deriving the underlying initial mass function (IMF) is generally considered as an outstanding tool for revealing the physical mechanisms actually at play. However, it is only for a handful regions that the mass distribution has been derived crossing the sub-stellar boundary at $0.072 M_{\odot}$, (see Bouvier et al. 2003, and references therein). The mass function of young open clusters with an age of $\sim 100 \mathrm{Myr}$ seems best described by a lognormal law over the full mass range in a $\mathrm{d} N / \mathrm{d} \log M$ presentation, regardless of their exact age, metallicity or richness. On the contrary, young regions with active SF ( $\$ 3 \mathrm{Myr}$ ) may indicate a mutually discordant number of BDs per star (Luhman et al. 2003). Additionally the binary frequency among field BDs indicates that the formation of BD binaries cannot be a mere extension of the formation of binary low mass stars (Close et al. 2003). As argued by Kroupa \& Bouvier (2003), it would indicate that BDs do not follow the same general rules for formation as stars do. On the other hand, BDs are known to be accompanied by CS disks (Natta et al. 2002, 2004). Mohanty et al. (2005) specifically demonstrate that young BDs do experience a T Tauri-like phase. These findings combined with the recent detection of a wide ( $>200 \mathrm{AU}$ ) BD binary (Luhman 2004), and the similar velocity distribution for stars and BDs in synthetic small-N clusters (Delgado-Donate et al. 2004) all argue in favor of $\mathrm{BD}$ formation being very much similar to lowmass star formation. It is clear that the dominant process that determines the final mass of a low mass star or a BD is not settled upon.

Young open clusters that occupy the age interval of 10-50 Myr (hereafter called pre-main sequence clusters or PMS clusters) can provide the clues to the issues listed above. There are a number of reasons why this age interval is critical in deriving the sub-stellar IMF. Unlike SF regions, PMS clusters provide the sampling of a complete stellar population immediately after the end of the active star forming phase, thus revealing the full stellar product. Secondly BDs in PMS clusters are still bright and easily detected and in addition they do not suffer from prodigious and inhomogeneous extinction as is generally the case for SF regions. From a methodological point of view, the IMF is obviously not what is observed, but is what is obtained after transformation of the luminosity function. The derived IMF thus depends on the theoretical predictions for the dependence of magnitude on mass and age (and metallicity). Baraffe et al. (2002) emphasized that no current BD model can be relied upon for ages less than $1 \mathrm{Myr}$, due to uncertainties in the treatment of convection, mass accretion rate, and atmospheric parameters $\left(\mathrm{H}_{2}\right.$ abundance). It is therefore that mass functions derived for somewhat older regions, like PMS clusters that will play a more accurate role than the MF derived for young SF regions. Clusters older than $\sim 150 \mathrm{Myr}$ start to be significantly affected by dynamical evolution, leading to serious problems in deriving the present-day MF. An additional complicating factor for these somewhat older clusters is the intrinsically lower luminosities of BDs. Clusters in the PMS age interval are therefore well-suited to explore in a relatively unbiased way the MF and thus to constrain BD formation theories, provided that a large enough area is covered. Unfortunately,

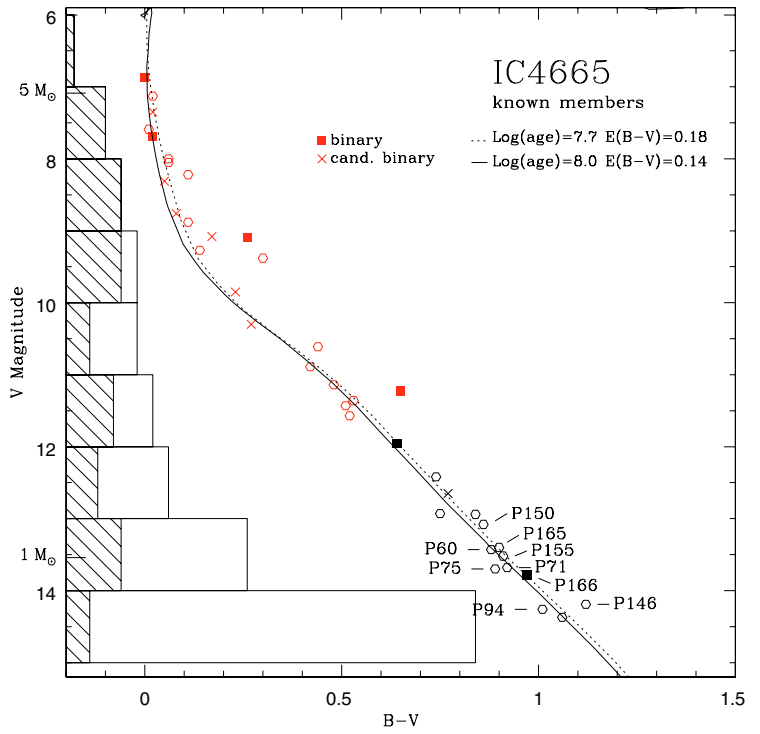

Fig. 1. Known member stars of IC 4665 . Two isochrones for $50 \mathrm{Myr}$ and $100 \mathrm{Myr}$ with indicated reddening (for $R_{v}=3.1$ ) from the Padua group (Girardi et al. 2000). Binary and candidate binary systems have specific symbols. Some members are named (see also Fig. 6). The plain histogram represents relative numbers in each magnitude bin of candidate members (Prosser 1993); the hatched part the confirmed members. The masses on the $y$-axis correspond to the $50 \mathrm{Myr}$ isochrone.

only a few PMS clusters do actually exist in the solar neighbourhood $(<500 \mathrm{pc})$.

IC 4665 is one of the few young open clusters within the solar neighbourhood, at a distance of $\sim 350 \mathrm{pc}\left(D_{\text {Hipp }}=385 \pm\right.$ $40 \mathrm{pc}$, Hoogerwerf et al. 2001). Previous work was undertaken primarily by Prosser and collaborators, scrutinizing candidate members on spectral type, Li-abundance, proper motion, radial velocity, $\mathrm{H} \alpha$-emission and X-rays with ROSAT (Prosser 1993; Prosser \& Giampapa 1994; Martin \& Montes 1997; Giampapa et al. 1998). An age estimate of IC 4665 by Mermilliod (1981) indicated an isochronal age of 30-40 Myr based on an analysis of the upper main-sequence stars. However Prosser (1993) argued that due to membership uncertainties, photometric errors and a colour effect of high rotational velocities, the upper main sequence stars are consistent with the age of $\alpha$ Per and may even indicate an age similar to the Pleiades. The age of IC 4665 is therefore not firmly established, but should lie somewhere between $30 \mathrm{Myr}$ and $100 \mathrm{Myr}$. By definition IC 4665 is possibly not a PMS cluster. The cluster sequence is well fitted both by a $50 \mathrm{Myr}$ and $100 \mathrm{Myr}$ isochrone (see Fig. 1). Although located out of the Galactic plane at a latitude of $b=+17^{\circ}$ a significant background stellar population does exist in that specific direction. We refer to Prosser (1993) for an overview of the work by various authors prior to 1990. In Fig. 1, we summarize current membership of IC 4665 prior to the work presented here in the form of a colour-magnitude diagram. The listed characteristics of IC 4665 combined with its rather well-known intermediate mass and solar-mass members render the cluster an excellent target for probing the IMF into the regime of very low mass stars and brown dwarfs. 
This paper is organized as follows. We give a brief overview of the observations, data reduction and calibration in Sect. 2. Section 3 describes the procedure for selecting new candidate members reaching well below the hydrogen burning limit. The fractional contamination by non-member objects is estimated in Sect. 4 using control fields, $K$-band photometry, and proper motion. We derive and discuss the radial distribution, the mass function and the total mass of the cluster in Sect. 5. A summary of the work presented is given in Sect. 6 .

\section{Observations and data reduction}

\subsection{Optical photometry}

As part of a CFHT large program, surveying star forming regions and young open clusters, IC 4665 was observed in the light of the $I$ (Mould) and $z$ filters. The telescope at that time was equipped with the $\mathrm{CFH} 12 \mathrm{~K}$ mosaic camera, the predecessor of the wide-field imager MegaCam. We refer to Moraux et al. (2003) for more details regarding observations, data reduction and astrometric calibration of the CFH12K. In what follows we describe some specific calibration steps applied to the IC 4665 set of observations.

The CFH12K camera consists of 12 individual $2048 \times$ 4096 CCDs each with angular scale of $0.206^{\prime \prime}$ pix $^{-1}$ arranged in a $2 \times 6$ mosaic (Cuillandre et al. 2001). In total $13 \mathrm{CFH} 12 \mathrm{~K}$ pointings (named field A to $\mathrm{M}$ ) centred on IC 4655 were observed for a total of 3.82 square degrees. Two control fields (C1 and C2), located at three degrees from IC 4665 at the same Galactic latitude were observed in order to estimate the foreground and background contamination of field stars. The central coordinate and the observation dates of each field are given in overview in Table 1. The coordinates of the fields were chosen such that each mosaic would overlap another. The overlaps are used to determine the external error on the photometry and allow the creation of an internal photometric system by calibrating the fields to one "master" field. The projection on the sky of the covered area relative to the brightest members of IC 4665 is shown in Fig. 2. Each of the 13 fields was observed for three different exposure times: $2 \mathrm{~s}, 30 \mathrm{~s}$ and $300 \mathrm{~s}$ or $360 \mathrm{~s}$ in $I$ and $z$ band. It allows the sampling of member stars for a range in masses between approximately 1 and $0.01 M_{\odot}$.

Objects were extracted from the CCD frames using the Sextractor software package (Bertin \& Arnouts 1996). To increase the accuracy on the photometric measurements, we used sextractor in conjunction with a point spread function (PSF) source extraction procedure. The PSF is determined for each individual CCD of the CFH12K mosaic for each field by the most well-behaved stars using the PSFex software (Bertin, priv. comm.; see also Kalirai et al. 2001). The procedure obviously requires a constant PSF profile across a CCD. A changing PSF profile however was observed on the I-band images for each CCD of the CFH12K mosaic and for each of the three exposures. The PSF profile was constant as function of $X$-coordinate, but changed as function of $Y$-coordinate: at $Y$-coordinates less than $\sim 500$ the PSF profile turns into an asymmetric, "teardrop"-like shape. This instrumental effect becomes especially apparent when comparing magnitudes
Table 1. Central coordinates of IC 4665 pointings (fields) with CFHT using $\mathrm{CFH} 12 \mathrm{~K}$ camera. Fields $\mathrm{C} 1$ and $\mathrm{C} 2$ are control fields.

\begin{tabular}{cccc}
\hline \hline Field & $\begin{array}{c}\text { RA }(2000) \\
(\mathrm{h}, \mathrm{m}, \mathrm{s})\end{array}$ & $\begin{array}{c}\text { Dec }(2000) \\
\left({ }^{\circ},{ }^{\prime}\right)\end{array}$ & Date \\
\hline A & $17: 46: 18$ & $+05: 43: 00$ & $19-5-2002$ \\
B & $17: 43: 38$ & $+05: 43: 00$ & $20-5-2002$ \\
C & $17: 48: 58$ & $+05: 43: 00$ & $20-5-2002$ \\
D & $17: 44: 58$ & $+06: 10: 00$ & $20-5-2002$ \\
E & $17: 48: 06$ & $+06: 07: 00$ & $20-5-2002$ \\
F & $17: 48: 00$ & $+05: 17: 00$ & $09-6-2002$ \\
G & $17: 44: 58$ & $+05: 13: 00$ & $09-6-2002$ \\
H & $17: 46: 18$ & $+06: 31: 00$ & $10-6-2002$ \\
I & $17: 47: 25$ & $+04: 53: 00$ & $10-6-2002$ \\
J & $17: 45: 00$ & $+04: 51: 00$ & $09-6-2002$ \\
K & $17: 43: 38$ & $+06: 31: 00$ & $09-6-2002$ \\
L & $17: 49: 25$ & $+06: 31: 00$ & $10-6-2002$ \\
M & $17: 42: 23$ & $+05: 17: 00$ & $09-6-2002$ \\
\hline C1 & $17: 51: 03$ & $+08: 05: 54$ & $12-6-2002$ \\
C2 & $17: 40: 53$ & $+02: 50: 14$ & $12-6-2002$ \\
\hline
\end{tabular}

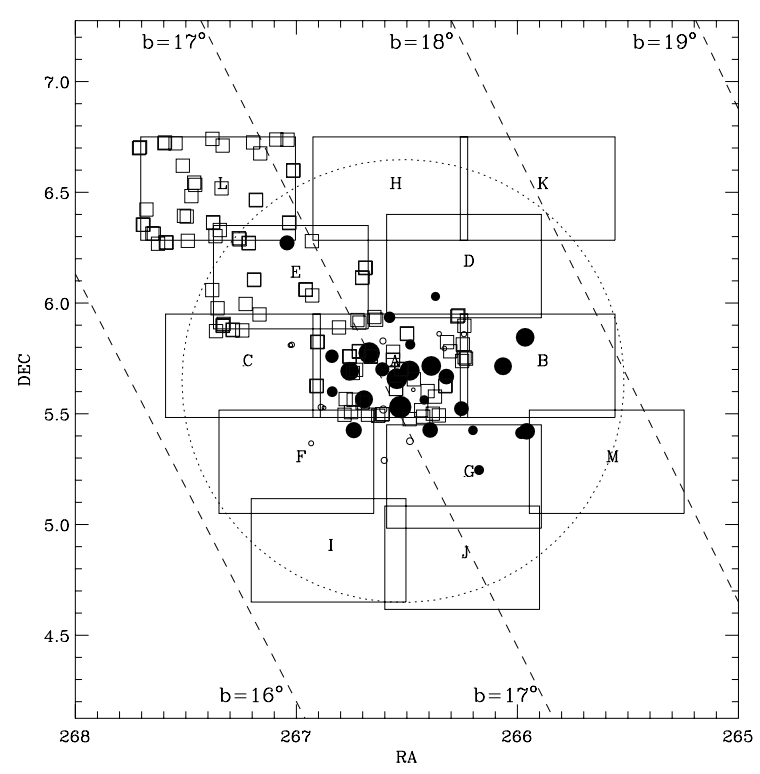

Fig. 2. Thirteen CFH12K fields (large boxes) covering 3.82 square degrees of IC 4665 were observed. Note the overlaps between the various fields. The small boxes correspond to the fields observed with CFHT-IR. IC 4665 members are represented by the large filled dots, whose sizes are indicative of their flux in $V$-band. The large dashed circle corresponds to a tidal radius for the best fit King profile (see Sect. 5).

obtained with aperture photometry and PSFex photometry. Such a comparison reveals an underestimation of the $I$-band magnitude and reaches a maximum $0.2^{\mathrm{m}}$ for PSF extracted stars at $Y$-coordinates less 50 . This is due to the loss of flux by the difference in shape of the actual stellar PSF and the applied model-PSF. Surprisingly, images obtained in the $z$-band do not suffer from variable PSF profile, hinting at an $I$-band 

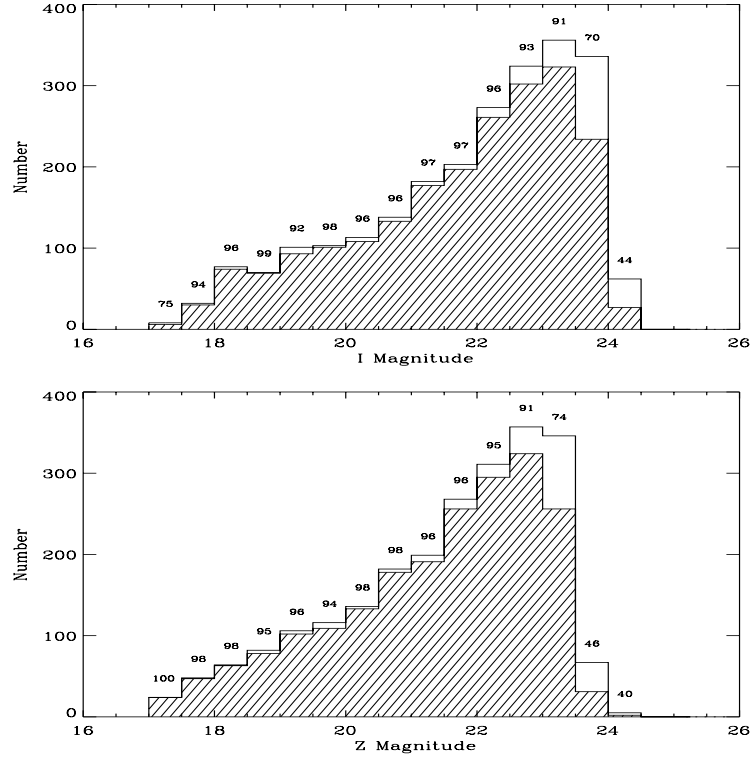

Fig. 3. Histogram of objects in field $\mathrm{D}$ located in the overlap with field $\mathrm{K}$. The hatched histogram presents objects detected in both fields, and measures the completeness at each magnitude bin.

filter problem of the $\mathrm{CFH}$ telescope. As far as we know, this is the first time this problem is reported for CFH12K I-band images. The immediate consequence of the variable PSF is that objects at low $Y$-coordinates on the CCD appear redder than they actually are. The PSF effect introduces therefore a higher fractional contamination of non-members to our photometrically selected candidate member catalogues, but does not lead to detection failure of IC 4665 members. On the other hand, magnitudes determined from PSF fitting are more accurate than aperture photometry. We therefore chose to continue using the PSF magnitudes, however flagging candidate member stars when found at low $Y$-coordinates. The completeness of the survey is exemplified in Fig. 3, after applying nominal zeropoints as determined by the CFHT's Elixir pipe-line (Magnier $\&$ Cuillandre 2004). These limits have been determined from the stars detected in the overlapping region of the $300 \mathrm{~s}$ exposure between fields $\mathrm{D}$ and $\mathrm{K}$. The figure shows that $I$ and $z$ band are $90 \%$ complete down to a magnitude of $\sim 23^{\mathrm{m}}$; the $I$-band is about $0.5^{\mathrm{m}}$ deeper than the $z$-band.

Some residual zeropoint offsets between the CCDs of the $\mathrm{CFH} 12 \mathrm{~K}$ mosaic were found to be present after cross correlating stars located in the overlapping regions (see Fig. 2). In Fig. 4 we show an example of the difference in instrumental magnitudes for the same stars observed in the overlapping region between CCD 06 of field I and CCD 11 of field J (30 s exposure). The upper part of the figure indicates the difference in $I$ and $z$ magnitude (first and second panel) and the considerable difference in the resulting $I-(I-z)$ CMD (third panel). For every overlap the photometric shift in $I$ and $z$-band was determined on a field-by-field and a CCD-by-CCD basis. In addition, an instrumental magnitude difference between the various exposures times was also found to occur. In this case we cross-correlated the stars taken at different exposure times to obtain the photometric shifts between $2 \mathrm{~s}, 30 \mathrm{~s}$ and $300 \mathrm{~s}$. In this way an internal calibration of the full dataset was achieved.

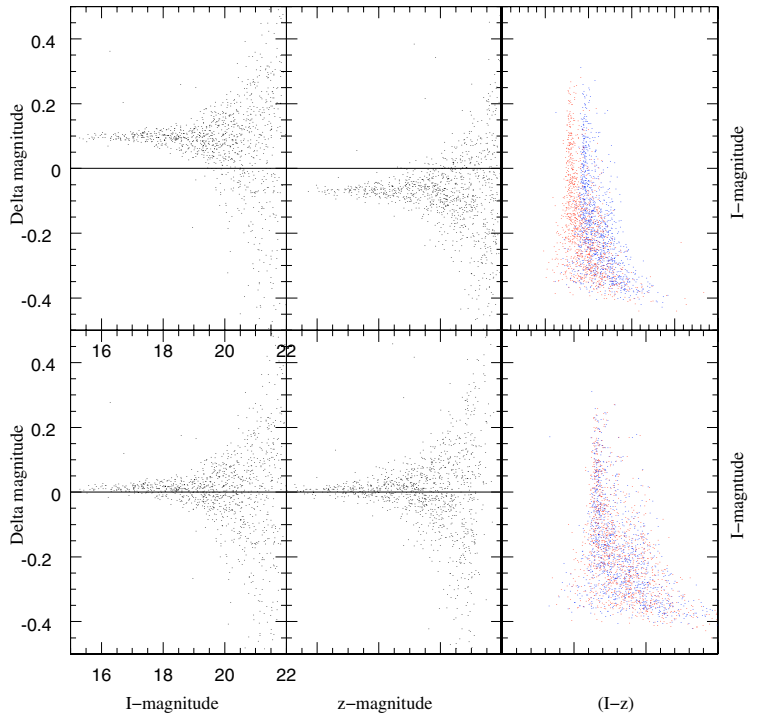

Fig. 4. Exemplifying the systematic shift between observations of the same part of the sky but with different CCDs of the CFH12K mosaic. The left ( $I$-band) and middle ( $z$-band) columns show CCD06 of field I minus CCD10 of field $\mathrm{J}$ in a $30 \mathrm{~s}$ exposure, before (upper row) and after (lower row) applying a zeropoint shift. The final effect in a CMD is shown in the right column.
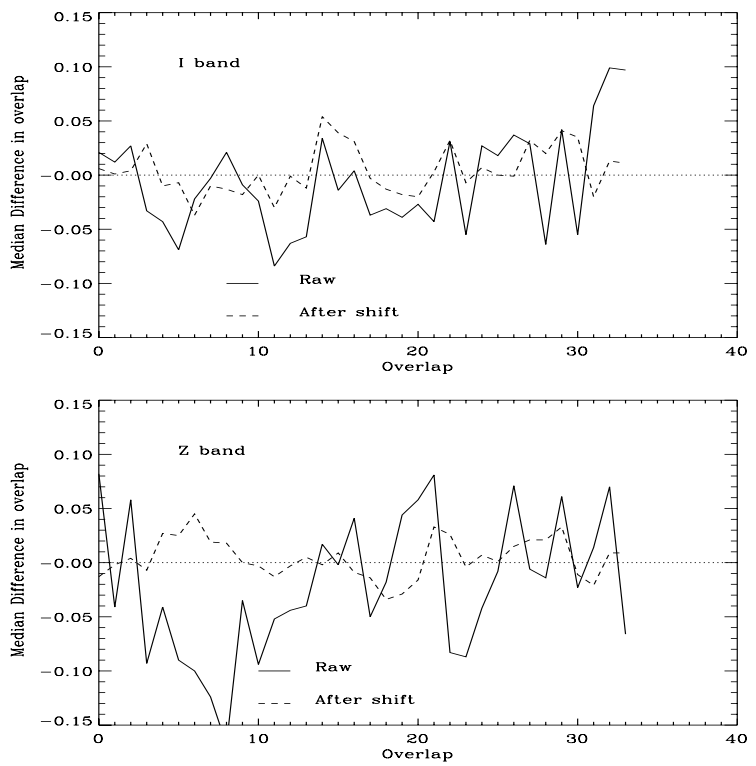

Fig. 5. The resulting median difference in I-band (top panel) and $z$-band (bottom panel) between the 34 overlapping regions of IC 4665 . The full line indicates the difference in photometry before the internal calibration ("raw"), the dotted line after applying a photometric shift. This shift has been derived from the overlapping regions, putting the photometry on one internal system.

The lower part of Fig. 4 exemplifies the improved photometric correspondence between CCD 06 of field I and CCD 11 of field $\mathrm{J}$ after the internal calibration. Figure 5 shows the median difference in $I$-band and $z$-band for all the 33 overlapping regions that were used in the internal photometric calibration. It shows the decreased differences between the photometry of the various overlaps. 


\subsection{Infrared photometry}

The next step in the derivation of IC 4665 properties after the optical selection of the new candidate members using the calibrated $I, z$ colour-magnitude diagram (see Sect. 3 ) is the step of weeding out interloper contaminants to the selected member dataset. $K^{\prime}$-band imaging of 101 optically selected IC 4665 candidate members was obtained with the $1 \mathrm{k} \times 1 \mathrm{k}$ CFHT IR camera (Starr et al. 2000) on July 10-12, 2003, and on May 30-June 3, 2004. The targets stars were chosen such as to probe the radial contamination factor, i.e. the stars observed with CFHT-IR were selected from either field A, E or L. All the observed fields with CFHT-IR are indicated in Fig. 2. The exposure time varied from 4 to $14 \mathrm{~min}$ for candidates with I magnitudes ranging from 17 to 22 . Each object was dithered on 5 to 7 positions on the detector, so that the median of the images would provide an estimate of the sky background during the exposure. Individual images were dome flat fielded, sky subtracted, then registered and added to yield the final $K^{\prime}$-band image. $K$-band photometric standards from Hunt et al. (1998) were observed every couple of hours each night. Aperture photometry was performed on candidates and photometric standards. For a few close visual binaries a large aperture was first used to estimate the system's total flux, and PSF photometry was performed to derive the flux ratio of the system components. The derived $K$-band photometric zero point is $22.86^{\mathrm{m}} \pm 0.03$ for both runs, assuming an average extinction coefficient of $0.07 \mathrm{mag} /$ airmass.

For the brighter optically selected candidate members, infrared data were obtained from cross-correlating all selected candidate members with the Two-Micron All-Sky Survey (2MASS) catalogues. The 2MASS catalogues are complete down to $K_{\mathrm{s}} \sim 14.3^{\mathrm{m}}$ with an uncertainty of $0.05^{\mathrm{m}}$. The completeness limit corresponds to a mass of $M \sim 0.20 M_{\odot}$ at the estimated age and distance of IC 4665.

\section{IC 4665 cluster member selection}

\subsection{Binary sequence}

The photometric selection of new members relies on the accurate positioning of the isochrone corresponding to the age and distance of IC 4665 in the optical I, z CMD. Throughout the paper we use evolutionary tracks calculated by Baraffe et al. (1998) and Chabrier et al. (2000) (the NEXTGEN and DUSTY models) unless otherwise stated. In this subsection we use a total of 10 known members (see Fig. 1) that have reliable $I$ and $z$ photometry in order to provide the actual positioning of the isochrone for an efficient member selection; the remaining IC 4665 members are saturated in the $I$ and $z$ images. A close look at the 10 faintest IC 4665 members in a NIR CMD (Fig. 6) using the Two Micron All-Sky Survey data indicate that their distribution is split in two groups. One half of the ten member stars is thus suspected to be binary objects. The reality of the binary sequence is supported by the star P 166 (filled square), a double-line spectroscopic binary (Prosser \& Giampapa 1994). Surprisingly, a binary sequence is not obvious from the optical CMD presented in Fig. 1, where we have indicated the names of the 10 members in question. The cluster isochrone for the



Fig. 6. The NIR CMD for IC 4665 members with confident $I, z$ photometric measurements (large symbols), based on 2MASS photometry. Small symbols are member stars without accurate $I, z$ magnitudes. Star P 166 is a binary. The figure shows that 5 out of 10 objects are candidate binary stars.

selection of new members which is presented in the next subsection is thus positioned in the $I, z \mathrm{CMD}$ on the presumably five non-binary members.

\subsection{Newly selected candidates}

In Fig. 7 we present the isochronal selection of new candidate members of IC 4665. For reference, brown dwarfs have an expected $I$ magnitude of $18.8^{\mathrm{m}}$ or dimmer at the estimated age and distance of the cluster. Candidate members are selected when their $I, z$ photometry locates them to the right of the $100 \mathrm{Myr}$ isochrone (full line) down to a magnitude of $I=22^{\mathrm{m}}$, if their PSF profile was found to be stellar. This age is a conservative estimate and in accordance with the isochronal age of the more massive members as presented in Fig. 1. The upper magnitude limit ensures the completeness of selected members down to an equivalent mass of $\sim 30 M_{\text {Jup }}$. The selection strategy therefore ensures the inclusion of all members, given the uncertainties. The ten member stars described in the previous paragraph are depicted by the large symbols at the top of the figure. The $100 \mathrm{Myr}$ isochrone is positioned to go through the bluest confirmed members of the single star sequence. The isochrone actually envelopes also quite well the probable members which are indicated by the small crosses. The presence of a binary sequence is also confirmed by the CFH12K I, $z$ photometry.

Figure 7 in fact shows the $100 \mathrm{Myr}$ isochrones (full lines) of the NEXTGEN models (Baraffe et al. 1998) and DUSTY models (Chabrier et al. 2000). For reference the $50 \mathrm{Myr}$ (dotted lines) isochrones are drawn as well. DUSTY model calculations include the settling of dust in the upper atmospheres of cool objects, which is not included in the NEXTGEN models. The transition between the dusty atmosphere and gaseous 


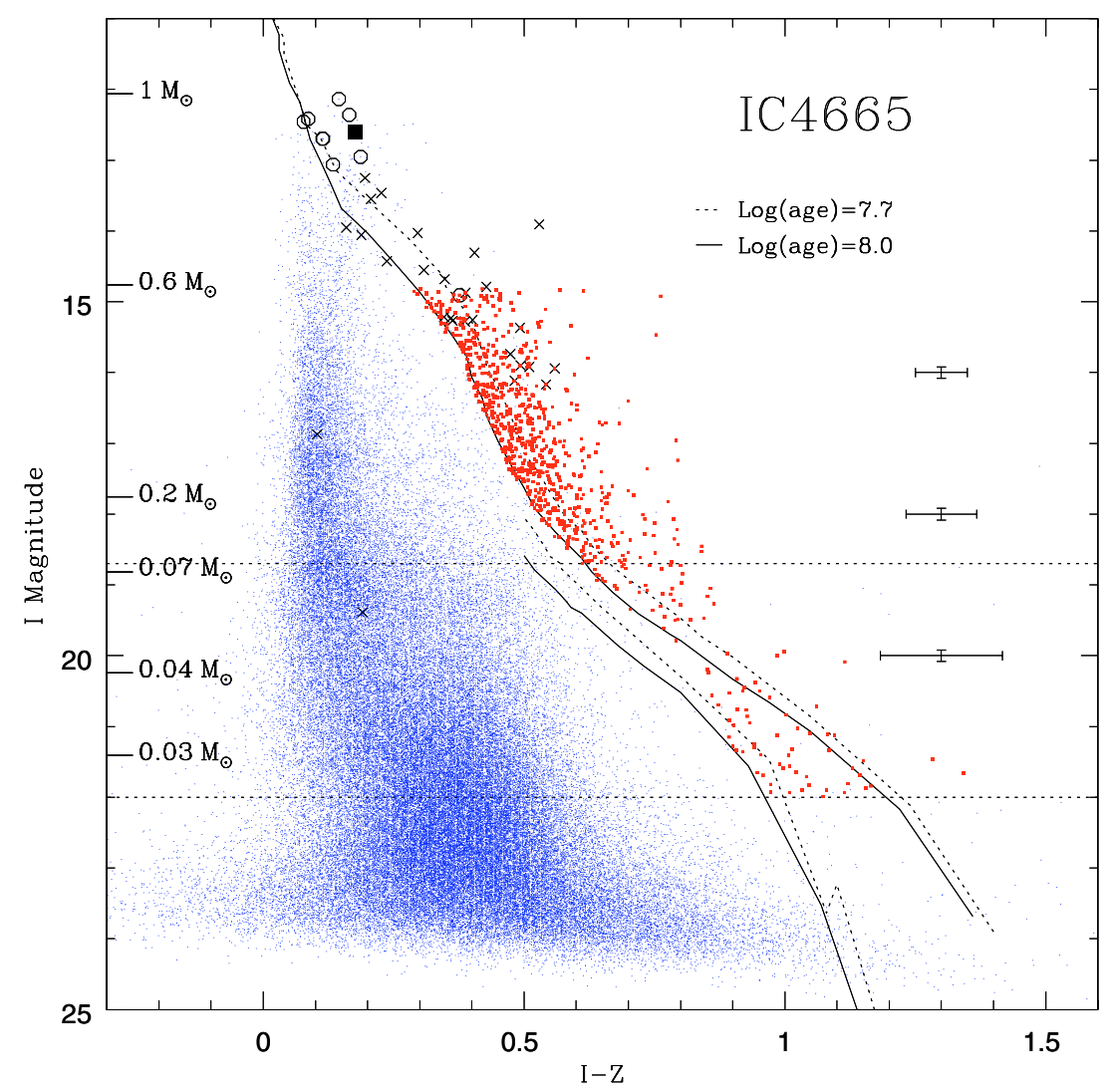

Fig. 7. Colour magnitude diagram of CFHT I, z observations for IC 4665. Some 94 Brown Dwarf candidate members and 691 low mass stellar candidate members have been photometrically selected using the theoretical isochrone corresponding to $100 \mathrm{Myr}$, down to a magnitude of $I=22^{\mathrm{m}}$. Member stars are given as circles or as a square (the confirmed binary P 166). Probable member stars identified by P92 are given as small crosses. Error bars have been determined from the overlap regions, with $Y$ (pixel) $>300$. Note that for clarity the Galactic field is plotted corresponding to two CFH12K fields, whereas all new candidate members are represented. The mass scale corresponds to the $50 \mathrm{Myr}$ NEXTGEN model for $>0.2 M_{\odot}$ and to the 50 Myr DUSTY model for lower masses.

atmosphere is thought to occur at $(I-z) \simeq 0.85$ corresponding to a NEXTGEN $T_{\text {eff }}$ of $3000 \mathrm{~K}$. This is the reason for the artificial "hook" in the selected objects at this colour. We especially note that had we chosen only NEXTGEN models for selecting candidate members of the lowest mass and not the DUSTY models, then only a very small amount of BDs candidates would have been found.

At the distance of IC 4665, the $100 \mathrm{Myr}$ isochrone intersects the foreground and background main sequence and giant branch stars in the CMD at about $0.8 M_{\odot}$, rendering the selection procedure at these masses highly inefficient. For clarity, Fig. 7 does not show all the stars extracted from the three exposures, but for two fields only (A and E). This translates to about one sixth of the total number of extracted stars in the surveyed area. On the contrary, all the selected candidate members are plotted. The selected objects were further checked by eye to eliminate any spurious detection due to hot pixels and/or bad CCD columns. The selection procedure delivered a total of 94 brown dwarf candidate members $\left(18.8^{\mathrm{m}}<I<22^{\mathrm{m}}\right)$ from $300 \mathrm{~s}$ measurements, 529 low mass stars $\left(15.8^{\mathrm{m}}<I<\right.$ $18.8^{\mathrm{m}}$ ) measured in the $30 \mathrm{~s}$ exposure and 163 low mass stars $\left(14.8^{\mathrm{m}}<I<15.8^{\mathrm{m}}\right)$ measured in the $2 \mathrm{~s}$ exposures. A list of these objects is found in Table 3 (available only at the CDS).

\section{Contamination by foreground and background stars}

The contamination in the foreground by faint M-dwarfs and by highly reddened bright objects behind the cluster in the direction of IC 4665 is anticipated to be quite substantial. The large numbers of newly selected candidate members makes this obvious. In this section we try to quantify the statistical contamination fraction and constrain memberships using $K$-band photometry and public proper motion data.

\subsection{Statistical contamination}

An estimate for the statistical contamination is derived from the two control fields. These fields are located well beyond the cluster boundary of IC 4665 . The same selection procedure to identify candidate members as explained in the previous section was applied to the two control fields. In the low mass stellar range down to the hydrogen burning limit $\left(14.8^{\mathrm{m}}<\right.$ $\left.I<18.8^{\mathrm{m}}\right)$, we select $108(48+60)$ objects in the two control fields. This translates to an absolute contamination of $164 \pm 13$ stars per square degree. The surveyed area centred on IC 4665 equals effectively 3.82 square degrees, and we thus expect 
626 contaminating stars. We selected 691 IC 4665 candidate members and we thus find a statistical contamination fraction of $\sim 85 \%$. That is only 1 in 6 photometrically selected candidate member is expected to be an actual cluster member. We note that contamination is expected to increase for the brighter stars, as IC 4665 sequence intersects the Galactic field giant branch at $I<15^{\mathrm{m}}$. For example in the range $\left(14.8^{\mathrm{m}}<I<15.8^{\mathrm{m}}\right)$, i.e. the candidate members selected from the short exposure, we calculate $90 \%$ contamination. In the substellar domain, corresponding here to $18.8^{\mathrm{m}}<I<22^{\mathrm{m}}$, we estimated the background contamination from the $300 \mathrm{~s}$ exposures. In the control fields $(9+2) 11$ objects were selected from the $300 \mathrm{~s}$ exposure, i.e. $17 \pm 5$ per square degree. Therefore we expect to find $\sim 64$ contaminants. In the surveyed area a total of 94 substellar candidates are found in the $300 \mathrm{~s}$ exposures. We conclude that the statistical contamination of our photometric selection of $\mathrm{BD}$ candidate members is on the order of $70 \%$. A more sophisticated estimate of the fractional contamination as function of $I$-band magnitude bins is not reliable. The cluster signal does not reach a high enough $\mathrm{S} / \mathrm{N}$ per bin.

The relatively low efficiency in selecting new member object compared to the efficiency reported by Moraux et al. (2003) for a similar procedure of photometric selection for new Pleiades members can be attributed to a smaller (absolute) Galactic latitude ( $b=-23$ for Pleiades, $b=+17$ for IC 4665$)$. A second factor yielding a much higher fractional contamination is the larger distance to IC 4665 (350 pc against $125 \mathrm{pc}$ ) resulting in $2.2^{\mathrm{m}}$ difference in apparent magnitude. The IC 4665 stellar and substellar sequences are therefore much closer in magnitude to the bulk of the foreground and background stars in a CMD. This difference is only partially alleviated by the younger age of IC 4665, as can be judged from the location of the isochrones corresponding to $50 \mathrm{Myr}$ and $100 \mathrm{Myr}$ in Fig. 7.

\subsection{Contamination estimate from K-band photometry}

A first step in actually removing contaminant objects is by using $K$-band photometry. Although from first principles cluster BDs and stars are expected to be only intrinsically more luminous than field stars of the same effective temperature (Zapatero Osorio et al. 1997), IR photometry does effectively separate out a fraction of non-member objects as shown in the case of $\alpha$ Per (Barrado y Navascués et al. 2001). In part the explanation may be sought in photospheric differences, not large enough to produce a significantly different $(I-z)$ colour, but that do become significant in the $(I-K)$ colour. This is exemplified in the optical-NIR CMD of Fig. 8. The small dots and open/filled asterisks represent all the selected stars based on the $(I-z)$ CMD, for which $K$-band photometry is available, observed by either 2MASS or with CFHT-IR as described in Sect. 2.2. A fraction of the $(I-z)$ colour selected objects are separated out in the diagram, not being located near the appropriate isochrone. The $K$-band contamination as function of $I$-band magnitude displays two peaks of $\sim 35 \%$ contamination at $I$-band magnitude of $14^{\mathrm{m}}$ and $18^{\mathrm{m}}$, i.e. the loci where the isochrones intersect the bulk of the field population.

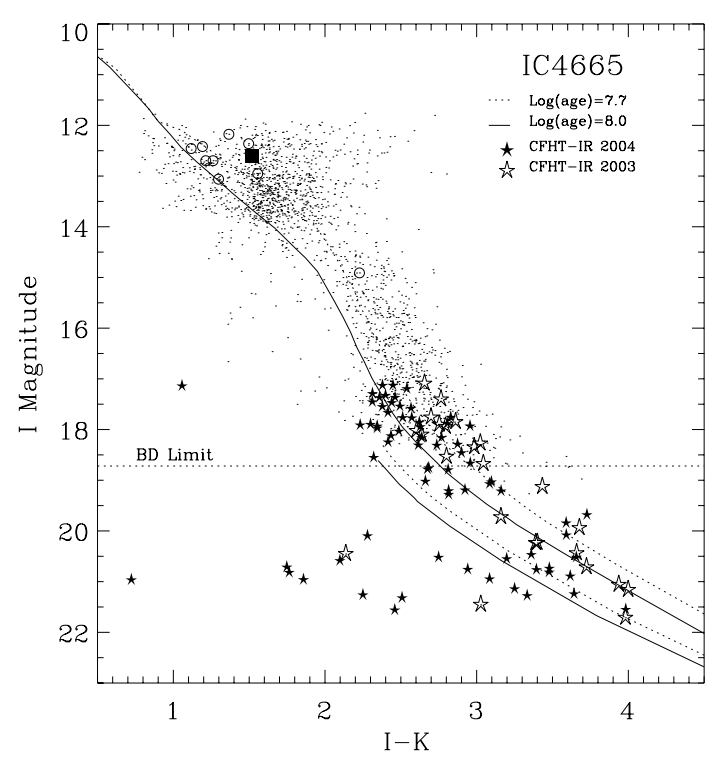

Fig. 8. Separating contaminant stars from optically selected objects using $K$-band photometry, either from the 2MASS survey (small dots) or observations with CFHT-IR instrument.

The correction step using $K$-band on the optically selection procedure has a relatively low efficiency for stars brighter than $I \sim 18^{\mathrm{m}}$. This can be judged from the 2MASS data in the NIR CMD of Fig. 8. At these magnitudes the $I-K$ colour allows at maximum the elimination of $35 \%$ per $I$-magnitude bin. A better estimate of the efficiency is to perform the same analysis using the control fields. Cross-correlating the optically selected objects in the two control fields (surely nonmembers) with the 2MASS dataset then we find that $80 \%$ of these objects are to the right of the cluster isochrone down to the $I \sim 18^{\mathrm{m}}$ in a $I,(I-K) \mathrm{CMD}$, and thus consistent with membership. For magnitudes fainter than $I \sim 18^{\mathrm{m}}$ using the CFHT-IR data, Fig. 8 shows that $K$-band photometry has an efficiency of $\sim 35 \%$. We see thus that $K$-band photometry does a reasonable job in separating out probable non-member stars from members. However the lower fractional contamination as determined from the $K$-band compared with the statistical estimate of the previous subsection directly implies that $K$-band is not the definitive answer to construct a clean sample of cluster members, at least not for the age, latitude and distance of IC 4665. Additional NIR photometry at other wavelength bands does certainly allow to constrain membership of potential candidate members that have initially been selected by making use of isochronal colours and magnitudes, as we'll see in the following section.

\subsection{Membership constraints from proper motion surveys}

The proper motion of IC 4665 is small and does not stand out significantly from the bulk field motion to be a strong constraint on membership. Hipparcos measured the astrometry of 13 IC 4665 members and obtained the following proper mo-

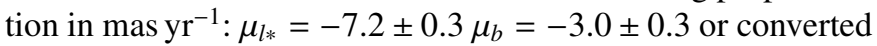
to equatorial coordinates $\left(\mu_{\alpha}, \mu_{\delta}\right)=(-0.4,-7.5) \mathrm{mas} \mathrm{yr}^{-1}$ 
(Hoogerwerf et al. 2001). Nevertheless, here we explore two public proper motion catalogues to evaluate membership for the optically selected candidate members, viz. the Tycho- 2 catalogue (Høg et al. 2000), and the USNO UCAC2 (Zacharias et al. 2004). The Tycho-2 catalogue is complete down to $K_{\mathrm{s}}=8^{\mathrm{m}}$, and provides proper motion with an accuracy of $2.5 \mathrm{mas} \mathrm{yr}^{-1}$. UCAC2 on the other hand is not complete for stars brighter than $R \simeq 8^{\mathrm{m}}$ due to saturation problems. For the stars in the R-band magnitude interval $13-16^{\mathrm{m}}$, UCAC2 provides proper motion with an error of $6 \mathrm{mas} \mathrm{yr}^{-1}$. Crosscorrelation with 2MASS All Sky Catalogue learns that 97\% of the 2MASS entries are in the UCAC2 catalogues for $K$-magnitude between $8-11^{\mathrm{m}}$.

We find 35 IC 4665 members in the UCAC2 catalogue for a mean proper motion of $\left(\mu_{\alpha}, \mu_{\delta}\right)=(0.0,-7.6)$ mas $\mathrm{yr}^{-1}$ with a 0.8 error in the mean value and a $\sim 5$ mas yr $^{-1}$ standard deviation in both the right ascension and declination proper motion distributions. The UCAC2 proper motion for IC 4665 is in good correspondence with the Hipparcos result of Hoogerwerf et al. The spread in proper motion space, i.e. the internal motion of IC 4665 can be judged from Fig. 9, in which the 35 member stars are indicated by the small open circles. Some 14 of these members are also found in Tycho-2. Their Tycho proper motions deviate from the UCAC2 results with $\left(\delta \mu_{\alpha}, \delta \mu_{\delta}\right)=$ $(3,3)$ mas $\mathrm{yr}^{-1}$, consistent within the uncertainties. The only discrepant result between the two catalogues we find for the visual binary $\mathrm{K} 67$, whose $\mathrm{UCAC} 2$ proper motion is not consistent with membership, but its Tycho-2 is.

Using UCAC2 to identify proper motion members to IC 4665, we require for selection that a star has a proper motion

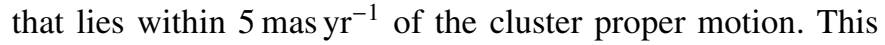
threshold is indicated by the large circle in Fig. 9. It equals the standard deviation in the UCAC2 proper motion of the member stars and this criteria will therefore exclude $32 \%$ of genuine cluster members. It is a compromise between optimising member selection and excluding background objects. We stress that proper motions deviant from the internal motion of the cluster as defined above is not a strong enough constraint to exclude membership.

In Fig. 10 we present the result of the proper motion selection in a CMD. The tiny dots are the proper motion selected candidate stars that are within the encircled area of the vector point diagram in Fig. 9. The cluster sequence is clearly recovered, and all member stars (open circles) have been plotted to indicate this. The small filled circles indicate all the rejected objects from the P93 catalogues. Note that on and near the cluster sequence for stars with a $K$-magnitude brighter than 10.5 (corresponding to about $1.2 M_{\odot}$ ) objects are located that do not have a counterpart in the P93 catalogues within a $2^{\prime \prime}$ cross-correlation radius, neither as a rejected nor as a confirmed member. Some among them are located within $1^{\circ}$ of the cluster centre, i.e. within the tidal radius of the cluster. These objects are clearly candidate IC 4665 members. The crosses in Fig. 10 identify the optically selected candidate members for which a UCAC2 proper motion exists. Figure 10 shows that currently public proper motion information for IC 4665 is limited to members brighter than $K \simeq 12^{\mathrm{m}}$ or equivalently to about $0.6 M_{\odot}$. The $(J-K)$ colour provides a strong constraint

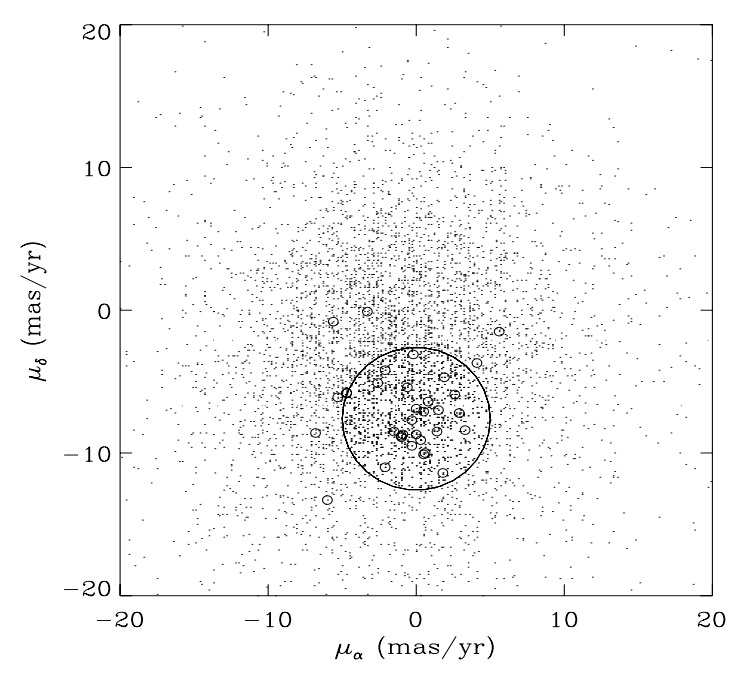

Fig. 9. A vector point diagram for stars within 1 degree from IC 4665, based on UCAC2 (Zacherias et al. 2004). The estimated bulk proper motion (circle) for IC 4665 based on the brightest stars using Tycho-2 data.

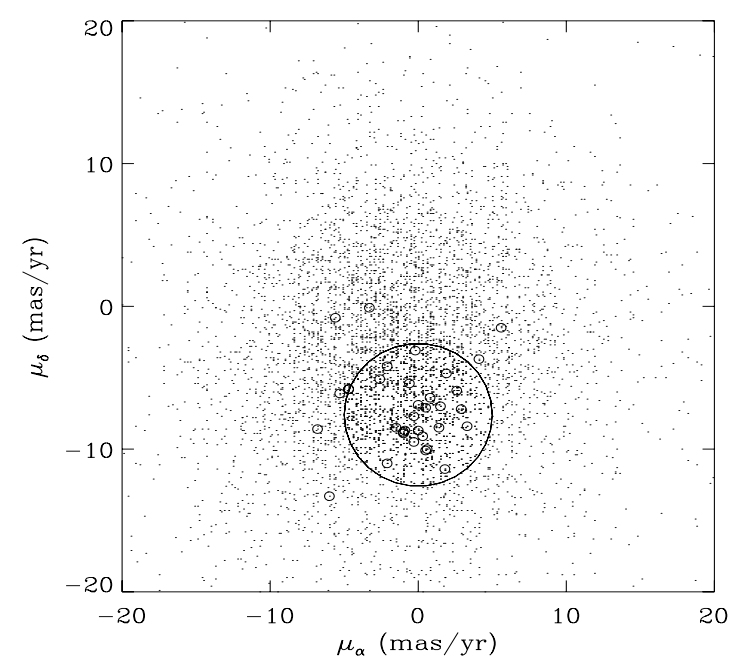

Fig. 10. The colours and magnitude for stars with proper motion consistent with IC 4665 (small dots), i.e. that are inside the circle of Fig. 9). Confirmed member stars (small circles) are added for reference. Small filled symbols are P93 non-members. Crosses are objects with UCAC2 proper motion that have been selected as candidate members in the optical survey.

for cluster membership in this case. Deep NIR observations in addition to the $K$-band are therefore strongly warranted. The new generation of NIR wide field cameras (WFCAM, WIRCAM) are especially designed to perform large surveys of young open cluster, e.g. like the Galactic Cluster Survey as part of the UKIDSS survey. For now we consider the stars that are too red in the upper of the diagram at color $J-K>0.6$ and $K<10.5^{\mathrm{m}}$ as likely non-members, and that the highest mass cluster stars are still on the main sequence. All eleven red giants except one that are brighter than $K<5.5^{\mathrm{m}}$ and lie outside the plotted range in Fig. 10 are confirmed non-members. 




Fig. 11. Histogram for stars with $K<10.5^{\mathrm{m}}$ and $J-K<0.6$. Plain histogram 2MASS All Sky Catalogue selected stars; single-hatched histogram all selected stars from P93 catalogues; double-hatched histogram confirmed members. Dashed line is the average background determined from control fields $\mathrm{C} 1$ and $\mathrm{C} 2$. Upper percentages: expected fraction of members, given the background level. Lower ratios: fraction of confirmed members to P93 selected candidates. See text for explanation.

\section{Discussion}

The radial and the mass distribution for members and candidate members are derived and discussed.

\subsection{The radial distribution for members and candidate members}

The radial dependence of the surface density in square degrees is derived for the high-mass member stars and the optically selected low-mass candidate members. For the latter we distinguish between stellar and BD candidates. To obtain these distributions, we first determine the mass-weighted central coordinates of IC 4665 using the high-mass members only. Adopting weights equaling the third power of the flux in the $V$-band magnitude, the cluster is found centred on $\mathrm{RA}(2000)=17^{\mathrm{h}} 46^{\mathrm{m}} 04^{\mathrm{s}}$, $\operatorname{Dec}(2000)=+05^{\circ} 38^{\prime} 53^{\prime \prime}$.

Three radial distributions for high-mass members are presented in Fig. 11. High-mass stars in this case are stars with a $K$-band luminosity smaller than $K=10.5^{\mathrm{m}}$ or equivalently a mass larger than $\sim 1.2 M_{\odot}$. This mass threshold corresponds to the location in a CMD where the cluster sequence intersects the background population (e.g. Fig. 7). We estimate the completeness of the cluster census as function of radius and the total radial extent of IC 4665 using both the P93 members and the unbiased 2MASS catalogues. From 2MASS we select highmass stars located within $1.5^{\circ}$ of the centre that have a colour bluer than $J-K=0.6$. The colour requirement excludes the foreground red GB and AGB stars, a population that makes up a large contaminant fraction at $K$-band luminosities $K<10.5^{\mathrm{m}}$ (see Fig. 10). The plain histogram with error bars in Fig. 11 depicts the 2MASS selected high-mass stars. The radial extent of IC 4665 is easily read off as the distribution is seen to attain background levels at radii larger than $\sim 1^{\circ}$. The dashed line denotes in fact the average surface density determined for a similar sized area centred on the $\mathrm{C} 1$ and $\mathrm{C} 2$ control fields. The control field surface density clearly marks the level of the stellar background level at the coordinates of IC 4665.

For comparison we added to Fig. 11 two radial distributions of P93 member stars: a single-hatched and a double-hatched histogram. They represent the surface density profile for all candidate members with $K<10.5^{\mathrm{m}}$ and $J-K<0.6$ and the census of confirmed high-mass members with the same colour and $K$-band magnitude constraints (29 objects), respectively. The candidate high-mass members have been investigated for cluster membership using various criteria in various studies. Among them there exist confirmed members, confirmed nonmembers and stars without definite classification (see Sect. 1). The bin at $1^{\circ}$ of the single hatched histogram contains zero confirmed candidates. This confirms the maximum extent of the cluster derived from 2MASS selected stars.

We note the ratios written above the bins of the plain and single-hatched histograms. The ratios of the plain histogram indicate the statistical fraction of cluster stars as function of radius, given the background level (dashed horizontal line). The ratios on the single-hatched histogram indicate the number of confirmed high-mass members to high-mass candidates. The two ratios at each bin are in good agreement, indicating that the current knowledge of the relative number of high-mass member stars of each bin is a good representation of the actual relative number. If the radial distribution of cluster members is best-described by a tidally truncated King profile (King 1962), we can thus fit and compare the profile of high-mass members with that of the low-mass constituency adopting a tidal radius of $1^{\circ}$. The bottom histogram of the left panel in Fig. 12 shows again the distribution for the high-mass members, i.e. the double-hatched histogram of Fig. 11. A by-eye fit to the first four radial bins points to a core radius $r_{\mathrm{c}}$ equaling $\sim 0.25^{\circ}$ (full line). In order to illustrate the uncertainty on this core radius, a King profile for $r_{\mathrm{c}}=0.5^{\circ}$ is also indicated by a dashed line. This profile still fits the observed distribution within the error bars. We consider it as the upper limit to the high-mass star core radius for IC 4665.

The upper histogram of the left panel in Fig. 12 shows the radial dependence of the optically selected candidate stellar members in the mass range of 0.07 and $0.6 M_{\odot}$. Error bars are equivalent to the statistical uncertainty for each bin. We note first that the surface densities derived for the control fields (shaded areas) are in good agreement with surface densities at radii larger than $r_{\mathrm{t}}=1.0^{\circ}$. This is the case for both the low-mass stars and the BD candadite members. It shows that IC 4665 cannot be much more extended on the sky, which gives confidence that the area covered with the CFH12K camera has been sufficient (see Fig. 2). The King profiles derived for the high-mass stars are scaled and plotted using the same line styles. A comparison between the best-fit high-mass King profile (full line) with the radial distribution of low-mass stars reveals that the two are not consistent. On the other hand a King profile with $r_{\mathrm{c}}=0.5^{\circ}$ (dashed line) is in reasonable correspondence. We thus see that the low-mass stellar population 

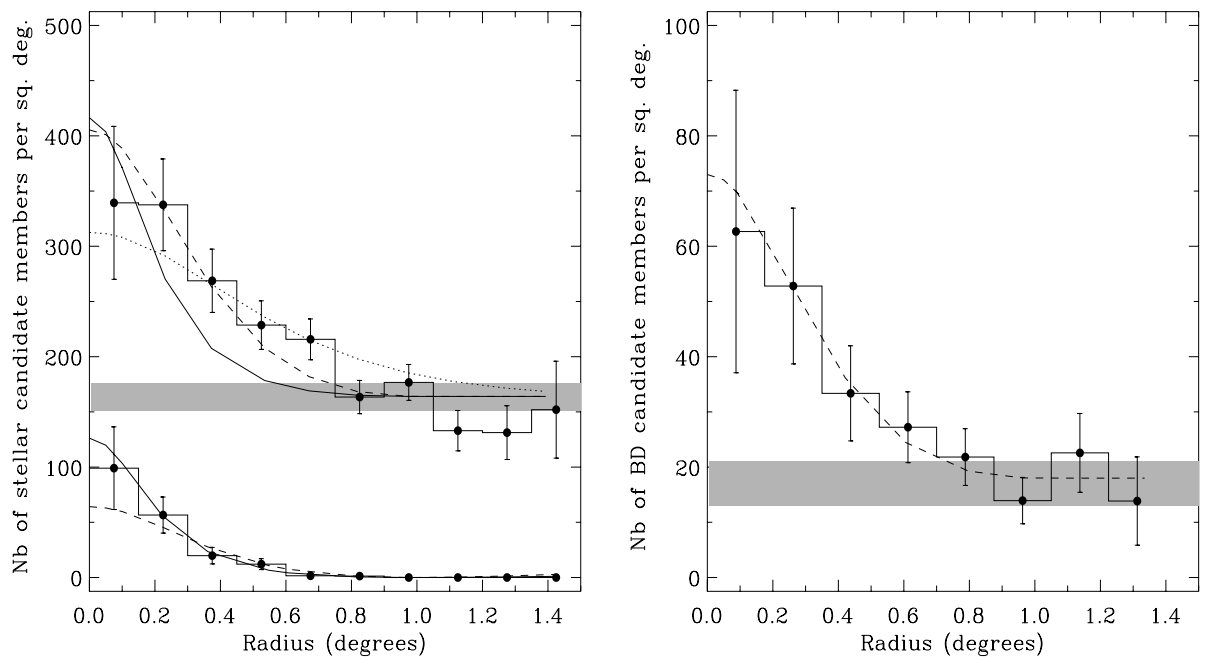

Fig. 12. Left: upper histogram presents the radial distribution for candidate members with $14.8^{\mathrm{m}}<I<18.8^{\mathrm{m}}$. Lower histogram equals the double-hatched histogram of Fig. 11. The shaded areas are the stellar background estimates derived from the control fields. Full lines are King profiles with $r_{\mathrm{c}}=0.25^{\circ}$ and $r_{\mathrm{t}}=1.0^{\circ}$; dashed lines King profiles with $r_{\mathrm{c}}=0.5^{\circ}$ and $r_{\mathrm{t}}=1.0^{\circ}$; dotted line is the scaled Pleiades low-mass profile with $r_{\mathrm{c}}=0.71^{\circ}$ and $r_{\mathrm{t}}=1.97^{\circ}$. Right: the radial distribution for the BD candidate members.

of IC 4665 has a $r_{\mathrm{c}} \gtrsim 0.5^{\circ}$. One can thus conclude that for $r_{\mathrm{c}}=0.5^{\circ}$ the surface densities of high-mass and low-mass stars are consistent within the statistical uncertainties. Nevertheless, it is probable that the low-mass stars have a broader distribution on the sky than the high-mass stars. This is illustrated by the dotted King profile in Fig. 12. It corresponds to the distribution for the low-mass stars of the Pleiades. Scaled to the distance of IC 4665 this profile is described by $r_{\mathrm{c}}=0.71^{\circ}$, and $r_{\mathrm{t}}=1.97^{\circ}$ and is not extremely far off the observed distribution of the low-mass candidate members of IC 4665 .

The right panel of Fig. 12 displays the BD candidate distribution for which only a profile with $r_{\mathrm{c}}=0.5^{\circ}$ is overlaid. This profile corresponds best to the observed distribution, the uncertainties are however large. Nevertheless a King profile fit with $r_{\mathrm{c}}$ of 0.25 (high-mass stars) or 0.71 (low mass stars in Pleiades) are not consistent with the observed distribution (not plotted). We conclude that the radial distribution of BD candidates is less certain than the one of the low-mass stars, however it seems more reminiscent to the low-mass star than the highmass star distribution. It would indicate a mixed BD and lowmass stellar population in IC 4665 . On the contrary the highmass stars may occupy a smaller area on the sky, which may constitute the first indication of present-day mass segregation in IC 4665. It is worth mentioning that mass segregation between stars of masses $>3 M_{\odot}$ and lower mass stars has been reported for the open cluster NGC 2547 (Littlefair et al. 2003; Jeffries et al. 2004). This cluster has an age that is probable only slightly less than the one in IC 4665, and the clusters are of comparable mass. Littlefair et al. and Jeffries et al. provide evidence in favour of a primordial origin for the mass segregation of NGC 2547, adding to other instances of inferred primordial mass segregations in case of more massive clusters (see Bonnell \& Davies 1998; de Grijs et al. 2002). Therefore the reality of mass segregation in IC 4665 between stars of $\gtrsim 1.2 M_{\odot}$ and lower mass stars is of considerable importance, but in need of confirmation.
Finally, using the King profiles for the low-mass stars and BDs candidates we can estimate their total number in the cluster. IC 4665 consists of $30 \pm 10$ BDs and $105 \pm 20$ low-mass stellar members, where the uncertainties are estimated from trying King profiles for various normalization factors. A background contamination within the tidal radius then proves to be $80 \pm 5 \%$ and $60 \pm 10 \%$ for low-mass stars and BDs respectively; within a radius of $0.55^{\circ}$ contamination factors of $65 \pm 5 \%$ and $40 \pm 15 \%$ are expected. These estimates also take into account the statistical uncertainties in the background level. From the radial distribution we estimate the fraction of BDs to total members in IC 4665. At the upper mass end there are 35 members with masses in the range $1<M / M_{\odot}<5$ and an estimated 39 stars between 1 and $0.6 M_{\odot}$, based on a Salpeter IMF. Taking into account the uncertainties we find a range of $10-19 \%$ for the fraction of IC $4665 \mathrm{BDs}$ (down to a mass of $\sim 0.03 M_{\odot}$ ) to total members. These factions are consistent with fractions found for other young open clusters for which such data are available, viz. 10-15\% for the Pleiades, NGC 2516, and Blanco 1 (see Moraux et al. 2005, and references therein). In this respect IC 4665 thus seems to be a "normal" young open cluster.

\subsection{The mass function}

The age of IC 4665 is not better determined than within a margin of nearly $40 \mathrm{Myr}$. We derive therefore in this section the mass function of IC 4665 assuming ages of $50 \mathrm{Myr}$ and $100 \mathrm{Myr}$ and the concomitant interstellar extinctions. Masses are assigned to low-mass objects by converting the I-band magnitudes using the NEXTGEN and DUSTY models. At overlapping magnitudes these two models do not assign the same mass for given magnitude. To overcome this, we made an interpolation between the two models at the expected transition effective temperature of $3000 \mathrm{~K}(I-z \simeq 0.85)$. For the high-mass bins we adopt the models without overshooting from the Padua group (Girardi et al. 2000) and convert the $K$-band 

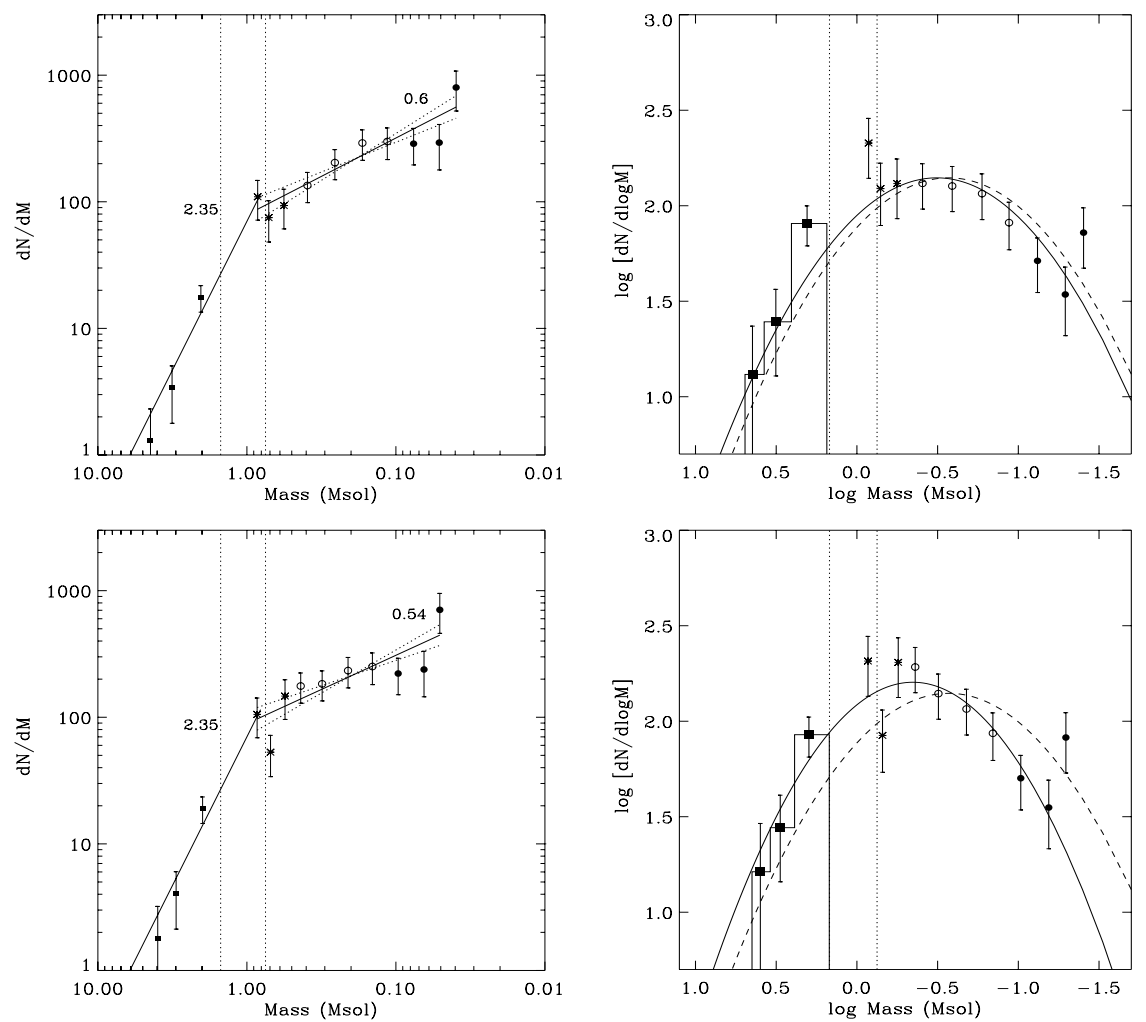

Fig. 13. The IC 4665 mass function in the $\mathrm{d} N / \mathrm{d} M$ (left column) and $\mathrm{d} N / \mathrm{d} \log (M)$ (right column); top row assuming an age of $50 \mathrm{Myr}$, bottom row for an age of $100 \mathrm{Myr}$. Symbols and lines in each panel have the same meaning. Squares denote candidate members within the tidal radius of $1.0^{\circ}$ selected from 2MASS, asterisks, open circles and filled circles from the $2 \mathrm{~s}, 30 \mathrm{~s}$ and $300 \mathrm{~s}$ exposures. The vertical dotted lines indicate the masses for which the cluster sequence intersects the background stellar population. Full lines in left column are Salpeter-law mass functions and power law functions (fits) with slopes of respectively -0.6 and -0.54 , and their associated $1 \sigma$ fitting uncertainties (dotted). In the right columns lognormal fits to the observed mass functions (full line) and the scaled Pleiades lognormal fit (dashed) are indicated (see text for details). Note the differences between the logarithmic mass functions for the two adopted ages.

magnitude to mass. We stress that in the derivation of the mass function presented here we do not incorporate any correction for the cluster depth ( $4 \%$ uncertainty on magnitude), undersampling of the cluster population due to various effects (hiding, bad pixels etc.) nor binarity (see e.g. Jeffries et al. 2004, for a discussion of these various effects).

The two mass functions for the two adopted ages of IC 4665 are presented in Fig. 13 in $\mathrm{d} N / \mathrm{d} M$ (Salpeter slope equals -2.35) and $\mathrm{d} N / \mathrm{d} \log M$ (Salpeter slope equals -1.35 ) representations. The 50 Myr mass function in numbers is presented in Table 2. Both mass functions correspond to candidate members located within the tidal radius of $1.0^{\circ}$ : high-mass stars (black squares, histogram) from 2MASS all sky catalogues applying the same colour and magnitude selection as in the previous subsection, and low-mass stars/BD candidate members from our CFHT survey. For the latter we use asterisk symbols, open circles and filled circles for candidate members found in $2 \mathrm{~s}, 30 \mathrm{~s}$ and $300 \mathrm{~s}$ exposures respectively. All mass bins have been statistically corrected for background contamination. The three high-mass bins from 2MASS are multiplied by $15 \%$. This factor has been derived using a 30 square degree region centred on control field $\mathrm{C} 2$. The low-mass bins are corrected using factors that are valid inside the tidal radius that have been estimated in the previous subsection for the low-mass and BDs candidates, viz. $20 \%$ and $40 \%$. Error bars correspond to statistical
Table 2. The mass function for IC 4665 for an age of 50 Myr. First column gives the central $I$-band magnitude of each bin. The column indicated with " $f$ " is the correction factor applied to account for the contamination by non-member objects.

\begin{tabular}{ccccrrr}
\hline \hline$I$ & $\begin{array}{c}\Delta M \\
\left(M_{\odot}\right)\end{array}$ & $N_{\text {obs }}$ & $\begin{array}{c}f \\
\%\end{array}$ & $N_{\text {corr }}$ & $\mathrm{d} N / \mathrm{d} M$ & $\mathrm{~d} N / \mathrm{d} \log M$ \\
& & \multicolumn{1}{c}{$\%$} & & & \\
\hline 13.30 & $0.92-0.77$ & 111 & 15 & 16.7 & 109.6 & 213.0 \\
14.30 & $0.77-0.66$ & 57 & 15 & 8.6 & 75.0 & 122.9 \\
15.30 & $0.66-0.47$ & 115 & 15 & 17.3 & 93.4 & 120.2 \\
16.18 & $0.47-0.31$ & 107 & 20 & 21.4 & 134.5 & 119.8 \\
16.93 & $0.31-0.20$ & 114 & 20 & 22.8 & 203.7 & 118.5 \\
17.68 & $0.20-0.135$ & 96 & 20 & 19.2 & 291.2 & 111.1 \\
18.43 & $0.135-0.093$ & 62 & 20 & 12.4 & 299.2 & 77.8 \\
19.23 & $0.093-0.059$ & 25 & 40 & 10.0 & 287.6 & 49.5 \\
20.08 & $0.059-0.044$ & 11 & 40 & 4.4 & 293.2 & 34.3 \\
20.93 & $0.044-0.035$ & 17 & 40 & 6.8 & 799.6 & 72.3 \\
\hline
\end{tabular}

uncertainties and the uncertainties in the above correction factors. Additional correction was made for the high-mass candidate members by applying the criteria reducing the nonmember objects that have been discussed in Sects. 4.2 and 4.3. We specifically note the vertical dotted lines in all four panels 
of Fig. 13. They indicate the masses for which the cluster sequence intersects the background stellar population. At these masses therefore a large relative number of non-member contaminants is expected, for which no specific correction is made.

The two mass functions in $\mathrm{d} N / \mathrm{d} M$ representation (left column of Fig. 13) are compared to a Salpeter mass function for mass bins $\gtrsim 1 M_{\odot}$, and find reasonable correspondence. More crucial is the shape of the mass function for bins $\lesssim 1 M_{\odot}$. For this mass range we performed an actual $\chi^{2}$-fit to the mass function, taking into account the 10 low-mass bins that represent the investigated mass range in this paper. For the $50 \mathrm{Myr}$ case (upper panel) we find a best linear fit for a slope of $-0.61 \pm 0.13$ for a $\chi^{2}=3.6$. We have indicated this fit by a full line, and the $1 \sigma$ uncertainties on the fit-parameters by the two dotted lines. For the $100 \mathrm{Myr}$ case (lower panel) the slope is less steep and the fit less good as can be judged from Fig. 13. We note that a power law relation with a slope of -0.6 is approximately the slope of the mass function for masses between 0.4 and $\sim 0.1 M_{\odot}$ found in other young open clusters (see e.g. Bouvier et al. 2003). We thus conclude that down to $\sim 0.1 M_{\odot}$, IC 4665 confirms this trend of the mass function.

For the three mass bins corresponding to the lowest masses for which the mass function crosses the H-burning limit the situation is more ambiguous. Either the mass function shows a constant -0.6 slope well into the substellar regime rendering two mass bins discordant, e.g. a dip at the stellar-substellar boundary as indicated by the linear fit; or on the other hand, the mass function flattens, rendering the last mass bin discordant. For both interpretations there exists supporting arguments.

In favour of the first interpretation is that such a dip in the mass function is a recurrent feature of young open cluster. It is discussed in detail by Dobbie et al. (2002) and suggested to be due to dust formation different from the dust formation recipe incorporated in the current generation of cool model atmospheres. It occurs at $T_{\text {eff }} \simeq 2700 \mathrm{~K}$, and reduces the object's luminosity at the investigated wavelengths. For the age of IC 4665 a dip in the mass function due to this effect is expected to be at $\sim 0.07 M_{\odot}$, i.e. corresponding to the observed dip in the left columns of Fig. 13.

The argument in favour of a flattening of the mass function in $\mathrm{d} N / \mathrm{d} M$, can be derived from the right-hand side column of Fig. 13. Here we present the mass function in the $d N / d \log M$ representation and we have fitted the mass function with lognormal functions. Lognormal functions are defined by an average quantity (mass in this case) and the width $(\sigma)$ of the lognormal distribution. A lognormal prescription is found to be a good representation for the mass function in the Pleiades (Moraux et al. 2003), Blanco 1 and NGC 2516. Moraux et al. (2005) reports for these three young open clusters a similar average mass of around $0.3 M_{\odot}$ and a similar $\sigma$ of $\sim 0.5$, producing quite a homogeneous picture of clusters in the age range 100-150 Myr. The case for IC 4665 is shown in the righthand column of Fig. 13. The top right panel corresponds again to a cluster age of $50 \mathrm{Myr}$ and the mass distribution is well fitted by a lognormal function with an average mass of $0.32 M_{\odot}$ (full line), except for the lowest mass bin. If one thus assumes that the complete mass function is described by a lognormal relation, then one expects the mass function to actually flatten in a $\mathrm{d} N / \mathrm{d} M$ representation, as is suggested by the mass bins at the H-burning limit on the left-hand side column of Fig. 13.

In Fig. 13, we also compare the lognormal fit of IC 4665 to the one of the Pleiades (with an $\bar{M}=0.27 M_{\odot}$, dashed line), and we note that they are quite similar. In the case of the somewhat younger cluster Blanco 1, Bouvier et al. (2005) actually find the same average mass as we deduce here for IC 4665. In fact the average mass of the Pleiades is suggestively bracketed by a higher average mass in younger clusters and lower average mass in the field (viz. 0.25 $M_{\odot}$, Chabrier 2003).

Finally we note, that despite the similar shape of the mass function in the $\mathrm{d} N / \mathrm{d} M$ representation (left column) for both adopted ages, the $\mathrm{d} N / \mathrm{d} \log M$ representations (right column) show a marked difference between the two ages. If IC 4665 were to be $100 \mathrm{Myr}$ old then a lognormal fit to the mass distribution tells us that the average mass of the cluster is significantly larger than found for clusters of such an age, viz. $0.45 M_{\odot}$. This would indicate that IC 4665 for some reason might have lost a substantial fraction of its lower mass constituent. In light of this we point out the remarkable coincidence in space (few degrees distance, where 1 degree is about $6 \mathrm{pc}$ ), age, and proper motion of IC 4665 with the young open cluster Collinder 359 for which we refer to Lodieu et al. (2005). We speculate that tidal fields between the two cluster could have stripped off preferentially the low-mass members of IC 4665.

Be this as it may, and assuming the younger age for IC 4665 we summarize that the mass function of IC 4665 be well approximated by a power law with slope -0.6 between 1 and $\sim 0.1 M_{\odot}$. The shape of the mass function of IC 4665 below the H-burning limit can either be explained in terms of the "M 7-8" gap as first described by Dobbie et al. (2002) producing a dip with respect to a linear relation, or alternatively, by assuming that mass functions in general are lognormal functions, in which case the mass bin corresponding to the lowest mass in Fig. 13 would be erroneous for a yet unknown reason. At the moment we cannot exclude either interpretation for the shape of the substellar mass function of IC 4665.

\subsection{The total mass and tidal radius}

In the previous subsection, we arrived at a tidal radius for IC 4665 of $\sim 1^{\circ}$ along various lines of analysis. The tidal radius depends amongst others on the total cluster mass and Galactic orbital radius of the cluster, a relation that was cast in the form of an equation for the Solar neighbourhood by Pinfield et al. (1998). We note that, as the authors point out, this equation is based on a simplified model. In the following we estimate the total mass of IC 4665 and confront this with the tidal radius. Under the assumptions that (1) the cluster census is complete for the high-mass stars down to $V<10^{\mathrm{m}}$ (which is likely to be an underestimate, see Fig. 1); and (2) a Salpeter-law holds between 5 and $0.7 M_{\odot}$ and a power law with slope -0.6 down to $0.01 M_{\odot}$, we find the total cluster mass of $\sim 300 M_{\odot}$. Here we accounted for an estimated $50 \%$ binarity with a flat q distribution. An upper limit of $<440 M_{\odot}$ to the cluster mass is furnished by assuming that all high-mass candidate member stars are actual members. An other and independent mass estimate can be 
found using 2MASS catalogues. Selecting blue $(J-K<0.6)$ high-mass stars within the tidal radius of IC 4665 and correct them for the background per $K$-band bin, one counts between 4.5 and $1.15 M_{\odot}$ a total of 56 stars. This translates to a total cluster mass of $350 M_{\odot}$, within the above upper limit. IC 4665 has thus a mass which is probably about half the mass of the Pleiades (Pinfield et al. 1998). Comparing now the cluster mass estimate with the tidal radius we find that IC 4665 does not follow the relation between cluster mass and tidal radius of Pinfield et al. (1998), their Eq. (12). In fact IC 4665 seems too small for the lowest of the above mass estimates by $0.5^{\circ}$. The mass and tidal radius can be reconciled in terms of the Pinfield relation if the distance of IC 4665 would have been underestimated by $200 \mathrm{pc}$. It would render the distance modulus $1 \mathrm{mag}$ larger, which seems unlikely (see Fig. 1). Alternatively tidal stripping of cluster members may also depend on the local environment of the cluster. We again point, as in the previous paragraph to the fact that IC 4665 is located in the Galactic field suspiciously close to the young open cluster Collinder 359 (Lodieu et al. 2005). We speculate that a small tidal radius for the total mass and a relatively large average mass for a cluster age of $100 \mathrm{Myr}$ could possibly be both the manifestation of ongoing tidal interactions and subsequent stripping of cluster members of IC 4665 and Collinder 359. Whether this process is really in effect certainly warrants a deeper investigation into the environment of these two young open clusters.

\section{Summary}

We have presented the photometric selection of 691 low-mass stellar and 94 BD candidate members to the young open cluster IC 4665. This extends the census of cluster (candidate) members well into the $\mathrm{BD}$ regime, going down to a few tens of Jupiter masses. We make an estimate of the contamination due to foreground and background objects in the field of IC 4665 on a statistical basis using control fields located at the same Galactic latitude. An additional selection procedure of contaminant objects for low-mass stellar and BD candidates is reported on the basis of public (2MASS) and new $K$-band photometry. Public proper motion survey data (Tycho-2, UCAC2) are also used to weed out candidate members for the somewhat brighter objects. Despite the substantial background contamination (up to $85 \%$ taken over the full surveyed area) the cluster does clearly stand out on the sky from the background population. This is the case for the brighter objects, but also the lower mass stars and BDs clearly show an increased number surface density towards the centre of the cluster. We study the radial distribution of various cluster populations and find a consistent tidal radius of $1^{\circ}$. There could be an indication of mass segregation, but this needs confirmation.

We find IC 4665 typical for a cluster in this age range with respect to (1) the fraction of $\mathrm{BD}$ (down to $\sim 0.03 M_{\odot}$ ) to total members, viz $10-19 \%$; (2) the slope of the mass function that it is well described by a power law with a power of -0.6 for the low-mass objects downto $\sim 0.1 M_{\odot}$; (3) a cusp in the mass function at about the Hydrogen burning limit, than can be explained in terms of the "missing" M 7-8 dwarfs, alternatively the cusp may be the product of an actual flattening of the mass function and a spurious value for the lowest mass bin, in concordance with the mass function being lognormal; (4) an average mass of $0.32 M_{\odot}$ when assuming an age of $50 \mathrm{Myr}$; (5) the width of $\sigma=0.5$ for the lognormal function fitted to the mass function in a logarithmic representation. However in two respects IC 4665 may stand out. We estimate the total cluster mass and find that it does not agree with the expected mass derived from the relation between tidal radius and total cluster mass (Pinfield et al. 1998). Secondly, we find that the average mass of IC 4665 is relatively large when assuming an age of $100 \mathrm{Myr}$. We speculate that increased stripping of member stars due to the interaction with the close-by young open cluster Collinder 359 could be the cause of these two effects. Clearly a better age determination for IC 4665 may resolve this issue. The analysis presented here adds in creating a consistent picture of the mass distribution in young open clusters. A rigorous evaluation of the mass function of IC 4665 using a large body of spectroscopic data will be the subject of paper II in our series on the young open cluster IC 4665.

Acknowledgements. We would like to thank E. Bertin for providing PSFex, I. Baraffe for computing isochrones for CFH12K filters, and E. Magnier for help in the derivation of photometric ZP. The research benefitted from financial assistance from the European Union Research Training Network "The Formation and Evolution of Young Stellar Clusters" (RTN1-1999-00436). This research has made use of the Simbad database, operated at the Centre de Donnees Astronomiques de Strasbourg (CDS), and of NASA's Astrophysics Data System Bibliographic Services (ADS). This publication makes use of data products from the Two Micron All Sky Survey, which is a joint project of the University of Massachusetts and the Infra-red Processing and Analysis Center/California Institute of Technology, funded by the National Aeronautics and Space Administration and the National Science Foundation. W.J.D.W. would like to thank the staff and students at the Osservatorio di Arcetri for their hospitality.

\section{References}

Baraffe, I., Chabrier, G., Allard, F., \& Hauschildt, P. H. 1998, A\&A, 337, 403

Baraffe, I., Chabrier, G., Allard, F., \& Hauschildt, P. H. 2002, A\&A, 382,563

Barrado y Navascués, D., Stauffer, J. R., Bouvier, J., \& Martín, E. L. 2001, ApJ, 546, 1006

Bertin, E., \& Arnouts, S. 1996, A\&AS, 117, 393

Bonnell, I. A., \& Davies, M. B. 1998, MNRAS, 295, 691

Bouvier, J., Moraux, E., Stauffer, J. R., Barrado y Navascués, D., \& Cuillandre, J. 2003, in IAU Symp., 147

Chabrier, G. 2003, PASP, 115, 763

Chabrier, G., Baraffe, I., Allard, F., \& Hauschildt, P. 2000, ApJ, 542, 464

Close, L. M., Siegler, N., Freed, M., \& Biller, B. 2003, ApJ, 587, 407

Cuillandre, J., Luppino, G., Starr, B., \& Isani, S. 2001, in SF2A-2001:

Semaine de l'Astrophysique Française, 605

de Grijs, R., Gilmore, G. F., Johnson, R. A., \& Mackey, A. D. 2002, MNRAS, 331, 245

Delgado-Donate, E. J., Clarke, C. J., \& Bate, M. R. 2004, MNRAS, 347,759

Dobbie, P. D., Pinfield, D. J., Jameson, R. F., \& Hodgkin, S. T. 2002, MNRAS, 335, L79

Giampapa, M. S., Prosser, C. F., \& Fleming, T. A. 1998, ApJ, 501, 624 
Girardi, L., Bressan, A., Bertelli, G., \& Chiosi, C. 2000, A\&AS, 141, 371

Høg, E., Fabricius, C., Makarov, V. V., et al. 2000, A\&A, 355, L27

Hoogerwerf, R., de Bruijne, J. H. J., \& de Zeeuw, P. T. 2001, A\&A, 365,49

Hunt, L. K., Mannucci, F., Testi, L., et al. 1998, AJ, 115, 2594

Jeffries, R. D., Naylor, T., Devey, C. R., \& Totten, E. J. 2004, MNRAS, 351,1401

Jiang, I., Laughlin, G., \& Lin, D. N. C. 2004, AJ, 127, 455

Kalirai, J. S., Richer, H. B., Fahlman, G. G., et al. 2001, AJ, 122, 257

King, I. 1962, AJ, 67, 471

Kroupa, P. 2001, MNRAS, 322, 231

Kroupa, P., \& Bouvier, J. 2003, MNRAS, 346, 369

Lin, D. N. C., Laughlin, G., Bodenheimer, P., \& Rozyczka, M. 1998, Science, 281, 2025

Littlefair, S. P., Naylor, T., Jeffries, R. D., Devey, C. R., \& Vine, S. 2003, MNRAS, 345, 1205

Lodieu, N., Bouvier, J., James, D. J., et al. 2005, A\&A

Luhman, K. L. 2004, ApJ, 614, 398

Luhman, K. L., Stauffer, J. R., Muench, A. A., et al. 2003, ApJ, 593, 1093

Magnier, E. A., \& Cuillandre, J.-C. 2004, PASP, 116, 449

Martin, E. L., \& Montes, D. 1997, A\&A, 318, 805
Mermilliod, J. C. 1981, A\&A, 97, 235

Mohanty, S., Jayawardhana, R., \& Basri, G. 2005, ApJ, 626, 498

Moraux, E., Bouvier, J., \& Clarke, C. 2005, Mem. Soc. Astron. It., 76, 265

Moraux, E., Bouvier, J., Stauffer, J. R., \& Cuillandre, J.-C. 2003, A\&A, 400, 891

Natta, A., Testi, L., Comerón, F., et al. 2002, A\&A, 393, 597

Natta, A., Testi, L., Muzerolle, J., et al. 2004, A\&A, 424, 603

Papaloizou, J. C. B., \& Terquem, C. 2001, MNRAS, 325, 221

Pinfield, D. J., Jameson, R. F., \& Hodgkin, S. T. 1998, MNRAS, 299, 955

Prosser, C. F. 1993, AJ, 105, 1441

Prosser, C. F., \& Giampapa, M. S. 1994, AJ, 108, 964

Reipurth, B., \& Clarke, C. 2001, AJ, 122, 432

Shu, F. H., Adams, F. C., \& Lizano, S. 1987, ARA\&A, 25, 23

Starr, B. M., Doyon, R., Beuzit, J., et al. 2000, in Optical and IR Telescope Instrumentation and Detectors, ed. M. Iye, \& A. F. Moorwood, Proc. SPIE, 4008, 999

Whitworth, A. P., \& Zinnecker, H. 2004, A\&A, 427, 299

Zacharias, N., Urban, S. E., Zacharias, M. I., et al. 2004, AJ, 127, 3043

Zapatero Osorio, M. R., Martin, E. L., \& Rebolo, R. 1997, A\&A, 323, 105 